\title{
Die Platzierung von verwahrten Personen in privaten Wohnheimen bei besonderer Pflegebedürftigkeit
}

Jonas Weber / Jann Schaub *

Vor allem eine restriktivere Entlassungspraxis führt dazu, dass sich im Justizvollzug immer mehr verwahrte Personen finden, die aus gesundheitlichen Gründen oder aufgrund ihres Alters pflegebedürftig werden. Da die herkömmlichen, staatlichen Justizvollzugsanstalten deren Betreuung in der Regel nur unzureichend gewährleisten können, stellt sich die Frage, ob verwahrte Personen aufgrund einer besonderen Pflegebedürftigkeit in privaten Wohnheimen untergebracht werden dürfen.

I. Einleitung 165

II. Überblick über die rechtlichen Grundlagen.................................................... 166

III. Art. 80 StGB: Abweichende Vollzugsformen ................................................. 169

1. Anwendbarkeit von Art. 80 StGB auf den Verwahrungsvollzug ................... 169

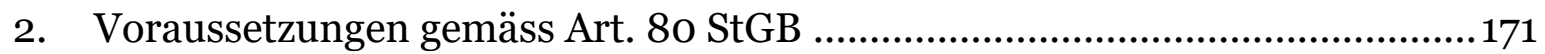

3. Anordnungszuständigkeit ................................................................ 173

IV. Verhältnis zu Art. 379 StGB....................................................................... 173

V. Verhältnis zu aktuellen parlamentarischen Vorstössen .................................. 174

VI. Fazit und Diskussion ............................................................................. 174

Zitiervorschlag: Jonas Weber/Jann Schaub, Die Platzierung von verwahrten

Personen in privaten Wohnheimen bei besonderer

Pflegebedürftigkeit, in: sui-generis 2018, S. 164

URL: $\quad$ sui-generis.ch/66

DOI: $\quad$ https://doi.org/10.21257/sg.66

* Jonas Weber (jonas.weber[at]krim.unibe.ch) ist Assistenzprofessor mit Tenure Track an der Universität Bern; Jann Schaub (jann.schaub[at]krim.unibe.ch) ist Oberassistent an der Universität Bern; Die beiden Autoren haben zur Thematik ein Kurzgutachten für die Bewährungs- und Vollzugsdienste des Kantons Bern, Amt für Justizvollzug, verfasst. 


\section{Einleitung}

1 Die Anzahl älterer Personen im Schweizer Justizvollzug nimmt zu. Damit einhergehend steigt die Anzahl Personen, die aufgrund ihres fortgeschrittenen Alters in absehbarer Zeit und auf unbestimmte Dauer (besonders) pflegebedürftig sein werden. Diese Entwicklung eröffnet neue Problembereiche in der Vollzugspraxis. Namentlich verwahrte Personen, die - alters- oder gesundheitsbedingt - pflegebedürftig sind, stellen sowohl Einweisungsbehörden als auch Anstalten vor Herausforderungen. ${ }^{1}$

Der Verwahrungsvollzug ist ausgerichtet auf die Sicherung gefährlicher straffälliger Personen und gekennzeichnet durch eine lange, unbestimmte Dauer. Verwahrte Personen geraten daher wohl zum einen mit erhöhter Wahrscheinlichkeit in die Situation, dass eine (besondere) Pflegebedürftigkeit während des Vollzugs

Zur zunehmenden Anzahl älterer Personen im Schweizer Justizvollzug und den sich daraus ergebenden Herausforderungen etwa: Ueli Hostettler/Irene Marti/Marina Richter, Lebensende im Justizvollzug. Gefangene, Anstalten, Behörden, Bern 2016; Barbara Baumeister/Samuel Keller, Alt werden im Straf- und Massnahmenvollzug, Zürich 2011; Nicolas Queloz, Finir sa vie en prison: ou quand l'Etat restraint excessivement le droit auch choix de fin de vie, in: Eva Maria Belser/Bernhard Waldmann, Mehr oder weniger Staat?, Festschrift für Peter Hänni zum 65. Geburtstag, Bern 2015, S. 517-529; Ueli Graf, Alt, krank, eingesperrt, in: Queloz/Noll/von Mandach /Delgrande (Hrsg.), Verletzlichkeit und Risiko im Justizvollzug, Bern 2015, S. 131-140; Bruno Graber/Erich Hotz/Peter Holenstein, Senioren im Strafvollzug, Kriminalistik 2014, S. 750-758; Franz Riklin (Hrsg.), Alt werden und Sterben hinter Gittern. Eine neue Realität für den Vollzug, Bern 2014; info bulletin 2/2016 (Fokus: Lebensende im Justizvollzug) und 2/2006 (Fokus: Senioren in Haft); Amt für Justizvollzug Zürich, «Alt werden im Justizvollzug», Bericht der Projektgruppe z.Hd. der Amtsleitung Justizvollzug vom 28. Mai 2014, gekürzte Version vom 26. August 2015 . eintritt. Es ist zu vermuten, dass sowohl das Älterwerden an sich als auch die lange Dauer der Inhaftierung (Haftschäden) diese Wahrscheinlichkeit befördern. Zum anderen führen restriktive Sicherheitsvorkehrungen ${ }^{2}$ und fehlende adäquate Betreuungsangebote in herkömmlichen staatlichen Justizvollzugsanstalten 3 zuweilen dazu, dass verwahrte Personen trotz einer besonderen Pflegebedürftigkeit weiterhin im selben, ungeeignet gewordenen Vollzugssetting verbleiben müssen.

3 Die besondere Pflege, die in konkreten Einzelfällen notwendig ist oder in absehbarer Zeit voraussichtlich notwendig werden wird, kann momentan in den wenigsten staatlichen Justizvollzugsanstalten erbracht werden. 4 Im Gegensatz dazu stünden beispielsweise in privaten Wohnheimen (u.a. Alters- und Pflegeheime) durchaus Plätze zur Verfügung, in denen eine angemessene Pflege gewähr-

$2 \mathrm{Zu}$ Sicherheitsabwägungen im aktuellen kriminalpolitischen Klima etwa Karl-Ludwig Kunz/Tobias Singelnstein, Kriminologie, 7. Aufl., Bern 2016, S. 305 ff. Bsp.: Jährlich unter 5\% (bedingte) Entlassungen aus der Verwahrung (Bundesamt für Statistik, «Massnahmenvollzug: Entlassungen nach Art der Massnahme», Stand: 27. Juli 2017); Verdoppelung der durchschnittlichen Aufenthaltsdauer in einer Massnahme seit 2007 (Bundesamt für Statistik, «Massnahmenvollzug: Entlassungsart und Aufenthaltsdauer», Stand: 27. Juli 2017).

3 Auf den Punkt gebracht von Ueli Hostettler/Irene Marti/Marina Richter, Das Leben soll lebenswert bleiben. Die Zunahme älterer Gefangener wird sich tiefgreifend auf den Vollzugsalltag auswirken, in: info bulletin 2/2016, S. 4-12, 6: Ist der Gesundheitsdienst vor allem darauf ausgerichtet, kranke Gefangene punktuell oder für «wenige Tage in einem Krankenzimmer zu behandeln, brauchen ältere, meist polymorbide Gefangene andauernde, intensive medizinische und pflegerische Betreuung.»

4 Siehe etwa Amt für Justizvollzug Zürich (Fn. 2), S. $19 \mathrm{f}$. 
leistet werden könnte. 5 Es stellt sich daher die Frage, ob verwahrte Personen unter gewissen Voraussetzungen in private Wohnheime eingewiesen werden dürfen bzw. sollen, welche die notwendige Pflege sicherstellen können, wenn ihnen ansonsten - d.h. in staatlichen Justizvollzugsanstalten - kein geeigneter Platz angeboten werden kann. Dieses Vorgehen würde voraussetzen, dass die geltende Rechtslage eine Platzierung von besonders pflegebedürftigen Verwahrten in privaten Wohnheimen zulässt.

Im Rahmen eines 2017 erstellten Kurzgutachtens, auf dessen Ergebnissen der vorliegende Beitrag beruht, konnten die Autoren Einsicht in drei Fälle aus zwei Kantonen nehmen, in denen verwahrte Personen aufgrund ihrer Pflegebedürftigkeit in privaten Wohnheimen platziert wurden, wodurch eine notwendig gewordene pflegerische Betreuung auf Dauer realisiert werden sollte. Durch diese drei Fälle konnten die dargestellten Herausforderungen konkret und anschaulich nachvollzogen werden.

5 Im vorliegenden Beitrag wird hergeleitet, weshalb es u.E. in der Kompetenz der einweisenden Behörden liegen muss, besonders pflegebedürftige verwahrte Personen zu deren Gunsten in privaten Wohnheime unterzubringen, wenn keine geeigneten Plätze in staatlichen Justizvollzugsanstalten zur Verfügung stehen. Zudem werden die grundsätzlichen $\mathrm{Zu}-$ lässigkeitsvoraussetzungen für eine Unterbringung in einem privaten Wohnheim diskutiert. Die Zweckmässigkeit, die Vor- bzw. Nachteile und die Haftbe-

5 Vgl. nur etwa die Liste der privaten Einrichtungen im Gebiet des Nordwest- und Innerschweizer Strafvollzugskonkordats. dingungen des Verwahrungsvollzugs in privaten Wohnheimen bleiben in diesem Beitrag ausgeklammert.

\section{II. Überblick über die rechtlichen Grundlagen}

6 Der Umgang mit einer besonderen Pflegebedürftigkeit von verwahrten Personen weist grundrechtliche Komponenten auf. Abgesehen von Artikeln in der Bundesverfassung ${ }^{6}$ betreffen mehrere Bestimmungen in internationalen Konventionen und Empfehlungen den Verwahrungsvollzug. Dazu ist anzumerken, dass nicht alle Staaten ein zweispuriges Strafsanktionensystem kennen, wie es in der Schweiz mit den Strafen und den Massnahmen verankert ist. In internationalen Konventionen und Empfehlungen wird in der Regel nur der Begriff «Strafvollzug» verwendet, der in einem weiteren Sinn verstanden wird und auch den Massnahmenvollzug umfasst. Die Anwendbarkeit der Vorgaben der internationalen Übereinkommen und Empfehlungen ergibt sich einzig daraus, dass die betroffene Person aufgrund einer strafrechtlich relevanten Tat gerichtlich zu einer freiheitsentziehenden Strafsanktion verurteilt wurde. 7

7 Insbesondere folgende menschen- und grundrechtlichen Vorgaben sind $\mathrm{zu}$ beachten: Das Recht auf Freiheit und

6 Insbesondere: Art. 7 (Menschenwürde), 8 (Rechtsgleichheit), 10 (Recht auf Leben und persönliche Freiheit), 31 (Freiheitsentzug) der Bundesverfassung der Schweizerischen Eidgenossenschaft vom 18. April 1999 (BV, SR 101).

7 Jörg Künzli/Anja Eugster/Maria Schultheiss, Haftbedingungen in der Verwahrung. Menschenrechtliche Standards und die Situation in der Schweiz, Schweizerisches Kompetenzzentrum für Menschenrechte (SKMR), Bern, 15. Juni 2016, S. $14 \mathrm{f}$. 
Sicherheit (Art. 9 UNO-Pakt II 8 , Art. 5 EMRK9), das Verbot der Folter, der unmenschlichen oder erniedrigenden Behandlung oder Bestrafung und menschenunwürdiger Haftbedingungen (Art. 7 und 10 UNO-Pakt II, Art. 1 und 16 FoK ${ }^{10}$, Art. 3 EMRK) sowie ergänzende Vorgaben aus dem «Soft Law», namentlich die einschlägigen Empfehlungen des Ministerkomitees des Europarats (insbesondere die Europäischen Strafvollzugsgrundsätze), die Standards des Europäischen Komitees zur Verhütung von Folter und unmenschlicher oder erniedrigender Behandlung (CPT-Standards) und die einschlägigen Resolutionen der UNO-Generalversammlung. ${ }^{11}$

Internationaler Pakt vom 16. Dezember 1966 über bürgerliche und politische Rechte, (SR 0.103.2).

9 Europäische Menschenrechtskonvention, Konvention vom 4. November 1950 zum Schutz der Menschenrechte und Grundfreiheiten, (SR 0.101).

10 Übereinkommen vom 10. Dezember 1984 gegen Folter und andere grausame, unmenschliche oder erniedrigende Behandlung oder Strafe (SR 0.105).

11 Etwa Empfehlungen Rec(2014)3 (Gefährliche Täter und Täterinnen), Rec(2006)2 (europäische Strafvollzugsgrundsätze), $\operatorname{Rec}(2003) 23$ (Langzeitinhaftierte), $\operatorname{Rec}(98) 7$ (Gesundheitsfürsorge im Freiheitsentzug); CPT/Inf/E (2002) 1 - Rev. 2015 (CPT-Standards); Nelson Mandela Rules. Zum Ganzen Künzli/Eugster/Schultheiss (Fn. 6), S. 12-15; Stefan Bérard/Nicolas Queloz, Fin de vie dans les prisons en Suisse: aspects légaux et de politique pénale, jusletter vom 2. November 2015, Rz. 4 ff. jeweils m.w.H. Zum Begriff und zur Reichweite von «Soft Law» (im Schweizer Recht) etwa: Benjamin Brägger, in: Niggli /Wiprächtiger (Hrsg.), Basler Kommentar zum Strafrecht I, Art. 1-110 StGB, Jugendstrafgesetz, 3. Aufl., Basel 2013 (fortan: Bearbeiter/in, BSK-StGB I), N. 3 zu Art. 74 StGB; Ronc, Pascal, Lebenslanger Freiheitsentzug, Art. 3 EMRK und die Rolle von Soft Law, ex ante 1/2017, S. 67-76; Urteil des EGMR Mursic gg. Kroatien, Nr. 7334/13 vom 20. Oktober 2016; Urteil des Bundesgerichts 1B_369/2013 vom 26. Februar 2013 E. 3.2 jeweils m.w.H.
8 Aus den aufgeführten Vorgaben lässt sich allgemein ableiten, «dass eine verurteilte Person an einem Ort unterzubringen ist, der Haftbedingungen ermöglicht, die vor diesen Garantien stand halten».12 Liegen bei verwahrten Personen besondere, gesundheits- oder altersbedingte Pflegebedürfnisse vor, ist diesen bei der Ausgestaltung der Vollzugsmodalitäten angemessen Rechnung zu tragen. ${ }^{13} \mathrm{Im}$ Sinne dieser Vorgaben ist es zwar auch bei besonders pflegebedürftigen und damit besonders vulnerablen Gefangenen grundsätzlich zulässig, den Freiheitsentzug aufrechtzuerhalten; ${ }^{14}$ indes lediglich unter der Voraussetzung, dass die Haftbedingungen entsprechend angepasst werden. Es besteht mithin ein grundrechtlicher Anspruch von besonders pflegebedürftigen verwahrten Personen auf Vollzugsmodalitäten, die ihrer Pflegebedürftigkeit angepasst sind. Vollzugsmodalitäten, welche dieser besonderen Pflegebedürftigkeit auf Dauer nicht entsprechen, sind aus menschen- bzw. grundrechtlicher Sicht unrechtmässig. ${ }^{15}$

12 Künzli/Eugster/Schultheiss (Fn. 6), S. 62.

13 Dazu Künzli/Eugster/Schultheiss (Fn. 6), S. 64 f.; Bérard/Queloz (Fn. 11), Rz. 24 ff. und 65 ff. jeweils m.w.H. Bsp. aus der Rechtsprechung des Europäischen Gerichtshofs für Menschenrechte (EGMR): Urteile Mouisel gg. Frankreich, Nr. 67263/01, Price gg. Vereinigtes Königreich, Nr. 33394/96, Matencio gg. Frankreich, Nr. 58749/00, Farbtuhs gg. Lettland, Nr. 4672/02, Tekin Yildiz gg. Türkei, Nr. 22913/04 und Hüseyin Yildirim gg. Türkei, Nr. 2778/02.

14 Siehe etwa Bérard/Queloz (Fn. 11), Rz. 101; Urteil des EGMR, Gengoux gg. Belgien, Nr. 76512/11; Stefan Trechsel/Peter Aebersold, in: Trechsel/Pieth (Hrsg.), Schweizerisches Strafgesetzbuch, Praxiskommentar, 3. Aufl., Zürich/St. Gallen 2018 (fortan: Bearbeiter/in, PK), N. 5 zu Art. 8o StGB.

15 Bérard/Queloz (Fn. 11), Rz. 98-100 unter Hinweis auf Entscheid des EGMR, Pretty gg. Vereinigtes Königreich, Nr. 2346/02. Siehe etwa auch Rec(98)7 Ziff. 50 und 51; CPT-Standards, Ziff. 70. 
9 Die Bestimmungen des Bundesrechts und kantonale Bestimmungen (inkl. jene der Konkordate) müssen im Licht dieser grundrechtlichen Vorgaben verstanden bzw. ausgelegt werden.

Der Bund hat seine Gesetzgebungskompetenz im Bereich des Straf- und Massnahmenvollzugs (Art. 123 BV) bis anhin zurückhaltend ausgeübt, was den Kantonen viel Freiraum für eigene Regelungen belässt (Art. 123 Abs. 2 BV). Der Massnahmenvollzug - und damit insbesondere auch der Vollzug von Verwahrungen ist im Vergleich zum Strafvollzug bundesrechtlich (noch) weniger dicht geregelt:

11 Von den Bestimmungen unter dem Titel «Vollzug von Freiheitsstrafen und freiheitsentziehenden Massnahmen» betreffen Art. 75-89 StGB («Vollzug von Freiheitsstrafen») den Strafvollzug, Art. 74 StGB («Vollzugsgrundsätze») sowie Art. 91 f. StGB («Gemeinsame Bestimmungen») sowohl den Strafvollzug als auch den Massnahmenvollzug und einzig Art. 90 StGB («Vollzug von Massnahmen») spezifisch den Massnahmenvollzug. ${ }^{16}$ Zusätzlich enthält das dem Vollzugsrecht vorgelagerte Strafsanktionenrecht (Art. 34-73 StGB) Bestimmungen, denen spezielle Vollzugsregeln für die einzelnen Massnahmen zu entnehmen sind. Vollzugsregeln zur Verwahrung finden sich in Art. 64 Abs. 2-4, 64a, $64 b$ und 65 StGB. Auch sind die Vollzugsaspekte der allgemeinen Bestimmungen von Art. 56 und 57 StGB auf die Verwahrung anwendbar. Unter dem Titel

16

Dazu etwa: Botschaft vom 21. September 1998 zur Änderung des Schweizerischen Strafgesetzbuches (BBl 1999 1979), 2108; Künzli/Eugster/Schultheiss (Fn. 6), S. 15.
«Einführung und Anwendung des Gesetzes» bestehen zudem Organisationsbestimmungen bzw. Kompetenznormen, die für den Massnahmenvollzug relevant sind: So können die Kantone gemäss Art. 377 Abs. 2 StGB Abteilungen für besondere Gefangenengruppen führen, namentlich für Gefangene bestimmter Altersgruppen (lit. b) und für Gefangene, die intensiv betreut oder behandelt werden müssen (lit. c). Auch wird den Kantonen erlaubt, privat geführten Anstalten und Einrichtungen eine Bewilligung $\mathrm{zu}$ erteilen, Strafen in der Form der Halbgefangenschaft und des Arbeitsexternats sowie Massnahmen nach den Art. 59-61 und 63 StGB zu vollziehen (Art. 379 Abs. 1 StGB). Schliesslich ist der Bundesrat befugt, nach Anhörung der Kantone, auf Verordnungsstufe Bestimmungen über den Vollzug von Massnahmen an kranken, gebrechlichen und betagten Personen zu erlassen (Art. 387 Abs. 1 lit. c StGB). Von dieser Kompetenz hat der Bundesrat bis anhin jedoch keinen Gebrauch gemacht. ${ }^{17}$ Zudem könnte der Bundesrat gemäss Art. 387 Abs. 4 lit. a StGB versuchsweise und für eine beschränkte Zeit neue Vollzugsformen einführen oder gestatten sowie den Anwendungsbereich bestehender Vollzugsformen ändern.

12 Keine der aufgeführten, spezifisch den Vollzug von Massnahmen bzw. noch spezifischer einzig den Vollzug von Verwahrungen betreffenden Bestimmungen des StGB enthalten eine ausdrückliche Regelung für die Platzierung von (besonders) pflegebedürftigen verwahr-

17 Anstatt vieler: Bérard/Queloz (Fn. 11), Rz. 134. Vgl. Verordnung zum Strafgesetzbuch und zum Militärstrafgesetz vom 19. September 2006 (VStGB-MStG, SR 311.01). 
ten Personen in privaten Wohnheimen. Zudem hat der Bundesrat seine Verordnungskompetenz gemäss Art. 387 Abs. 1 lit. c StGB bis heute nicht genutzt und folglich insbesondere keine Bestimmungen über den Vollzug von Massnahmen an (besonders) pflegebedürftigen verwahrten Personen erlassen.

13 Auch in den Kantonen bestehen - soweit ersichtlich - keine (ausdrücklichen) Rechtsgrundlagen zu der hier interessierenden Frage. Einzig die Richtlinie des Strafvollzugskonkordats der Nordwestund Innerschweiz betreffend die Hafterstehungsfähigkeit ${ }^{18}$, die gemäss Ziff. 2 Abs. 1 auch für den Massnahmenvollzug gilt, enthält einen impliziten Hinweis. Unter dem Titel «Angepasster Vollzug infolge gesundheitlicher Beeinträchtigungen» wird in Ziff. 4 lit. e der Richtlinie statuiert, dass bei schweren gesundheitlichen Beeinträchtigungen als abweichende Vollzugsform im Sinne von Art. 80 StGB beispielweise die Einweisung in eine privat geführte Institution denkbar sei. Da eine besondere Pflegebedürftigkeit ein Fall von Hafterstehungsunfähigkeit sein kann, kann diese Richtlinie mindestens im Rahmen der Auslegung des Bundesrechts weiterhelfen.

\section{Art. 8o StGB: Abweichende Vollzugsformen}

14 Als Zwischenfazit kann festgehalten werden, dass einerseits weder im Bundesrecht noch im kantonalen Recht eine ausdrückliche Regelung für die Platzie-

18 Richtlinie des Strafvollzugskonkordats der Nordwest- und Innerschweiz betreffend die Hafterstehungsfähigkeit vom 25. November 2016 (SSED 17 $\left.7^{\text {ter }} .0\right)$ rung von besonders pflegebedürftigen verwahrten Personen in privaten Wohnheimen besteht, sich hingegen andererseits aus den menschen- und grundrechtlichen Vorgaben (und entsprechend auch aus den in Art. 74 StGB statuierten Vollzugsgrundsätzen ${ }^{19}$ ) ein grundrechtlicher Anspruch verwahrter Personen auf Vollzugsbedingungen ergibt, die an ihre besonderen Pflegebedürfnisse angepasst sind.

15 Dieser Widerspruch ist auflösbar: Mittels einer Auslegung der vorhandenen Gesetzesbestimmungen kann den menschenund grundrechtlichen Vorgaben entsprochen werden. Eine analoge Anwendung von Art. 80 StGB («Abweichende Vollzugsformen») auf den Verwahrungsvollzug und in der Folge als Rechtsgrundlage für die Platzierung einer besonders pflegebedürftigen verwahrten Person in einem privaten Wohnheim bietet sich als naheliegende und einleuchtende Lösung an.

\section{Anwendbarkeit von Art. 8o StGB auf den Verwahrungsvollzug}

In Art. 8o StGB sind abweichende Vollzugsformen zugunsten des Gefangenen geregelt, die aufgrund des Gesundheitszustands des Gefangenen erforderlich sind. Die Regelung findet sich bei den Bestimmungen zum Vollzug von Freiheitsstrafen. Hinsichtlich therapeutischer Massnahmen (Art. 59-61 StGB) leuchtet diese systematische Einordnung durchaus ein: Deren Vollzug soll ohnehin unter geeigneten, d.h. den Bedürfnissen

$19 \overline{\text { Vgl. Graf (Fn. 1), S. } 134 \text { f.: «Ohne entsprechende }}$ Vorkehrungen bezüglich Unterbringung, Betreuung, Behandlung und Pflege von Angehörigen vulnerabler Gefangenengruppen können die Vollzugsgrundsätze des Strafgesetzbuchs nicht eingehalten werden (Art. 74 und 75 StGB).» 
der gefangenen Person angepassten, Rahmenbedingungen und an geeigneten Vollzugsorten erfolgen. Eine Ausnahmebestimmung im Sinne von Art. 80 StGB scheint für therapeutische Massnahmen daher nicht notwendig. ${ }^{20}$ Dies gilt insbesondere auch für den Vollzug therapeutischer Massnahmen in Privatanstalten, da es in der Kompetenz der Kantone liegt, private Einrichtungen für den Vollzug von therapeutischen Massnahmen zuzulassen (Art. 379 StGB).

17 Für den Vollzug von Verwahrungen trifft die Feststellung, dass eine Regelung i.S.v. Art. 80 StGB nicht erforderlich wäre, hingegen nicht zu. ${ }^{21}$ Im Gegenteil gibt es gute Gründe für eine analoge Anwendbarkeit von Art. 80 StGB auf den Verwahrungsvollzug. So ist davon auszugehen, dass der Vollzug von Verwahrungen in der Regel in einer Strafanstalt stattfindet. 22 Wenn verwahrte Personen im selben Setting untergebracht sind wie Personen, die eine Freiheitsstrafe verbüssen, können sich bei ihnen aufgrund eines besonderen (Gesundheits)Zustands dieselben Probleme ergeben wie bei Personen im Strafvollzug. Folglich braucht es dasselbe Korrektiv, um bei Vorliegen besonderer (gesundheitlicher) Umstände von den üblichen Vollzugsmodalitäten abweichen zu können. Überdies könnte sich sonst aufgrund des

20 Siehe etwa Cornelia Koller, BSK-StGB I (Fn. 10), N. 5 zu Art. 80 StGB; Andrea Baechtold, Abweichende Vollzugsformen, in: Brägger (Hrsg.), Das schweizerische Vollzugslexikon. Von der vorläufigen Festnahme zur bedingten Entlassung, Basel 2014, S. 1-4, $1 \mathrm{f}$.

21 Siehe Baechtold (Fn. 20), S. 1 f.: Die Rechtsprechung werde zu prüfen haben, «ob es sich hierbei nicht um ein gesetzgeberisches Versehen handelt und eine Anwendung von Art. 8o StGB auf Verwahrte nicht dennoch zulässig sei».

22 Dazu etwa: Marianne Heer/Elmar Habermeyer, BSK-StGB I (Fn. 10), N. 128 zu Art. 64 StGB. per 2007 für den Verwahrungsvollzug eingeführten dualistisch-kumulativen Systems $^{23}$ die Situation ergeben, dass während des der Verwahrung vorangehenden Vollzugs der Freiheitsstrafe gestützt auf Art. 8o StGB eine abweichende Vollzugsform praktiziert wird, diese dann aber bei gleichbleibender - oder durch den Zeitablauf sogar verstärkter - Pflegebedürftigkeit beim Übertritt in den Verwahrungsvollzug abgebrochen werden müsste. Damit wäre der Insasse im Verwahrungsvollzug schlechter gestellt als im vorangehenden Vollzug der Freiheitsstrafe.

18 Diese Überlegungen stehen in Einklang mit Art. 90 StGB. In Art. 90 StGB werden Besonderheiten des Massnahmenvollzugs dadurch geregelt, dass bestimmte Regeln des Strafvollzugs für den Massnahmenvollzug modifiziert bzw. präzisiert werden. Dieses gesetzgeberische Vorgehen schliesst nicht aus, dass Bestimmungen des Strafvollzugs, die von diesen ausdrücklichen Modifikationen unberührt sind, analog auf den Massnahmenvollzug angewendet werden können. Vielmehr war es ein erklärtes Anliegen der 2007 in Kraft getretenen Revision des Allgemeinen Teils, dass die Regeln des Strafvollzugs grundsätzlich analog für den Massnahmenvollzug gelten. ${ }^{24}$ Aus diesem Grund wurde auch die Regelungsdichte bei den nur für den Massnahmenvollzug gültigen Bestimmungen bewusst gering gehalten. ${ }^{25}$ Es sind keine einleuchtenden Gründe ersichtlich, weshalb der Gesetzgeber die

23 Art. 64 Abs. 2 StGB. Dazu etwa Günther Stratenwerth, Schweizerisches Strafrecht. Allgemeiner Teil II: Strafen und Massnahmen, 2. Aufl., Bern 2006, S. $58 \mathrm{f}$.

24 Heer (Fn. 22), BSK-StGB I, N. 3 zu Art. 90 StGB.

25 Siehe BBl 19991979 (Fn. 16), 2108 f. 
analoge Anwendung von Art. 80 StGB auf den Verwahrungsvollzug (bewusst) hätte ausschliessen wollen.

Art. 80 StGB gilt somit, über den Strafvollzug hinaus, analog für den Verwahrungsvollzug. ${ }^{26}$ Dies ist insbesondere auch unter dem Gesichtspunkt zu vertreten, dass in jüngerer Zeit mit dem sog. Abstandsgebot vermehrt ganz generell besondere Vollzugsbedingungen für verwahrte Personen begründet bzw. verlangt werden und in diesem Sinne nicht eine Schlechterstellung verwahrter Personen, sondern vielmehr deren Besserstellung verglichen mit Personen im Strafvollzug - anzustreben ist. ${ }^{27}$

\section{Voraussetzungen gemäss Art. 80 StGB}

20 Gemäss Art. 80 Abs. 1 StGB darf unter besonderen Bedingungen, die in der betroffenen Person liegen - Gesundheitszustand (lit. a), Schwangerschaft bzw. Geburt (lit. b), gemeinsame Unterbringung von Mutter und Kleinkind (lit. c) -, von den allgemeinen Vollzugregeln zugunsten dieser Person abgewichen werden. Der Gesetzgeber wollte mit dieser Bestimmung im neuen Recht den «Vollzug in angepasster Form» ausdrücklich kodifizieren, der gemäss der Praxis des Bundesgerichts zu Art. 40 Abs. 2 aStGB unter altem Recht zulässig

26 Zumindest implizit wohl auch Künzli/Eugster/Schultheiss (Fn. 6), S. 65; Baechtold (Fn. 20), S. 1 f.; Amt für Justizvollzug Zürich (Fn. 1), S. 20 und 39; Urteil des Verwaltungsgerichts des Kantons Zürich VB.2015.00781 vom 13. September 2016 E. 2.3; Richtlinie betreffend die Hafterstehungsfähigkeit (Fn. 18). Seit der 3. Auflage (2018) des Praxiskommentars explizit auch Trechsel/Aebersold (Fn. 14), PK, N. 5 zu Art. 8o StGB.

27 Siehe etwa Künzli/Eugster/Schultheiss (Fn. 6), S. 66 f. m.w.H. war. ${ }^{28}$ Durch Art. 80 StGB wird den zuständigen Behörden ein Ermessensspielraum zugunsten der betroffenen Person eingeräumt, damit einzelfallweise von den allgemeinen bzw. üblichen Vollzugsregeln abgewichen werden kann, wenn dies geboten scheint. Die Bestimmung bezweckt damit eine über die im Gesetz vorgesehenen Vollzugsformen hinausgehende Möglichkeit der Differenzierung und Individualisierung und ermöglicht dadurch in Einzelfällen angepasste Lösungen. ${ }^{29}$

21 Für die hier besprochene Thematik ist die über den Gesundheitszustand begründete Abweichung (lit.a) von den Vollzugsregeln relevant. Lehre und Rechtsprechung gehen diesbezüglich von einem weiten Gesundheitsbegriff aus, der neben schweren, chronischen Krankheiten auch Altersgebrechen und körperliche Behinderungen umfasst. Dabei sind die Ursachen des Gesundheitszustandes unerheblich, so kann dieser bspw. auch selbstverschuldet herbeigeführt worden sein. 30

22 Liegt ein derartiger Gesundheitszustand vor, kann die zuständige Behörde gestützt auf Art. 80 StGB mittels verschiedener Vollzugsmodifikationen reagieren:

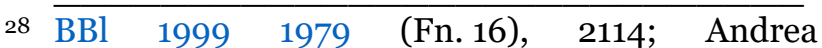
Baechtold/Jonas Weber/Ueli Hostettler, Strafvollzug, Straf- und Massnahmenvollzug an Erwachsenen in der Schweiz, 3. Aufl., Bern 2016, S. 145 N. 64. Siehe BGE 103 Ib 184; 106 IV 321.

29 Joe Keel, in: Hansjakob/Schmitt/Sollberger (Hrsg.), Kommentierte Textausgabe zum revidierten Strafgesetzbuch, 2. Aufl., Luzern 2006 (fortan: Bearbeiter/in, Kommentierte Textausgabe), S. 89; Antwort Bundesrat auf Anfrage 15.1073 Gross; Urteil des Verwaltungsgerichts des Kantons Zürich VB.2016.00448 vom 14. November 2016 E. 3.1.

30 Siehe etwa Trechsel/Aebersold (Fn. 14), PK, N. 3 zu Art. 8o StGB; Koller (Fn. 20), BSK-StGB I, N. 11 zu Art. 8o StGB; BGE 136 IV 97. 
Es ist bspw. denkbar, die betroffene Person von bestimmten Pflichten zu entbinden, die Zellentüre in der Nacht nicht zu verschliessen oder erleichterte Kontakte mit der Aussenwelt zu erlauben. ${ }^{31}$ Es ist aber ausdrücklich auch die Unterbringung in einer anderen geeigneten Einrichtung (Abs. 2) möglich. Das Gesetz konkretisiert dabei nicht, welche Einrichtungen als geeignet i.S.v. Art. 80 StGB gelten. Der Botschaft des Bundesrats folgend wird der Begriff der geeigneten Einrichtung in der Lehre indes weit ausgelegt. Als mögliche Vollzugsorte werden bspw. Spitäler, psychiatrische Kliniken, Entzugskliniken, Alters- und Pflegeheime, Wohnheime für AidsKranke und Heime einer religiösen Gemeinschaft genannt. Der Begriff umfasst unbestrittenermassen auch private Institutionen, namentlich private Wohnheime. 32

Die Anordnung abweichender Vollzugsformen setzt demnach zum einen voraus, dass die Beeinträchtigung des Gesundheitszustands - vorliegend die besondere Pflegebedürftigkeit - eine gewisse Intensität erreicht. Eine alters- oder krankheitsbedingte Pflegebedürftigkeit einer verwahrten Person genügt dem in Art. 80 StGB vorausgesetzten Gesundheitszustand jedenfalls dann, wenn sie eine gewisse Schwere und Dauer aufweist.

24 Zum anderen ist vorausgesetzt, dass dieser Beeinträchtigung unter normalen

Koller (Fn. 20), BSK-StGB I, N. 11 zu Art. 80 StGB.

32 Siehe BBl 19991979 (Fn. 16), 2115; Antwort Bundesrat auf Anfrage 15.1073 Gross; Koller (Fn. 20), BSK-StGB I, N. 18 zu Art. 80 StGB; Baechtold/Weber/Hostettler (Fn. 28), S. 145 N. 63. Vgl. Ziff. 4 lit. e der Richtlinie betreffend die Hafterstehungsfähigkeit (Fn. 18).
Vollzugsbedingungen nicht angemessen begegnet werden kann. Eine abweichende Vollzugsform gemäss Art. 80 StGB ist mit anderen Worten nur dann zulässig, wenn die erforderliche Pflege bzw. medizinische Betreuung im konkreten Einzelfall im Rahmen des regulären Vollzugs nicht (mehr) gewährleistet werden kann (sog. Hafterstehungsunfähigkeit).33 Dies bedeutet u.E., dass immer zuerst Alternativen innerhalb des Regelvollzugs, d.h. innerhalb einer Strafanstalt, geprüft werden müssen. U.E. wäre im Sinne des subsidiären Charakters von Art. 80 Abs. 2 StGB weiter auch die Möglichkeit der Verlegung in eine Abteilung für besondere Gefangenengruppen i.S.v. Art. 377 Abs. 2 StGB oder in eine Massnahmenvollzugsanstalt zu prüfen.

25 Zudem muss vor der Platzierung einer verwahrten Person in einem privaten Wohnheim selbstverständlich auch geprüft werden, ob die Person trotz der eingetretenen besonderen Pflegebedürftigkeit weiterhin als derart gefährlich zu beurteilen ist, dass ihre bedingte Entlassung gemäss Art. 64a StGB nicht in Betracht kommt.34 Ebenso muss die Umwandlung der Verwahrung in eine stationäre therapeutische Massnahme (Art. 65 Abs. 1 StGB) der Unterbringung der verwahrten Person in einem Wohnheim vorgehen, wenn die Umwandlung nicht aufgrund fehlender schwerer psychischer Störung, fehlender Therapierbarkeit oder fehlender Motivierbarkeit zu einer Therapie ausgeschlossen ist. Auch gegenüber regulären Vollzugsöffnungen i.S.v. Art. 90 Abs. $2^{\text {bis }}$ StGB (Wohn- und

33 Urteil des Verwaltungsgerichts des Kantons Zürich VB.2016.00448 vom 14. November 2016 E. 3.1. m.w.H.

34 Dazu etwa das Urteil des Bundesgerichts 6B_1198/2016 vom 29. Juni 2017. 
Arbeitsexternat) verhalten sich die abweichenden Vollzugsformen im Sinne von Art. 80 StGB subsidiär.

Erst wenn feststeht, dass die im Einzelfall der besonderen Pflegebedürftigkeit angemessenen Vollzugsbedingungen in staatlichen Justizvollzugsanstalten nicht gewährleistet werden können, kommt in analoger Anwendung von Art. 80 Abs. 2 StGB die Platzierung einer verwahrten Person in einem Wohn- bzw. Pflegeheim in Frage. Kann dem besonderen Pflegebedürfnis einer verwahrten Person auch mit einer Einweisung in eine "andere geeignete Einrichtung» i.S.v. Art. 80 Abs. 2 StGB nicht entsprochen werden, kann wiederum subsidiär eine Unterbrechung des Verwahrungsvollzugs (Art. 92 StGB) zur Anwendung kommen. 35

\section{Anordnungszuständigkeit}

27 Die Zuständigkeit für die Bewilligung abweichender Vollzugsformen muss sich aus dem kantonalen Recht ergeben, da die Kompetenz bundesrechtlich nicht geregelt ist. ${ }^{6}$ Besteht im kantonalen Recht keine entsprechende ausdrückliche Zuständigkeitsnorm, fallen Abweichungen von den Vollzugsformen innerhalb einer Institution in die Zuständigkeit der Institutionsleitung und die Einweisung in eine andere Einrichtung in die $\mathrm{Zu}$ -

$3 5 \longdiv { \text { Koller (Fn. 20), BSK-StGB I, N. } 1 1 \text { zu Art. } 9 2 }$ StGB; Trechsel/Aebersold (Fn. 14), PK, N. 5 zu Art. 8o StGB; Baptiste Viredaz/ André Vallotton, in: Roth/Moreillon (Hrsg.), Commentaire Romand, Code pénal I, Art. 1-110 CP, Basel 2009 (fortan: Bearbeiter/in, CR), N. 2 zu Art. 8o StGB; Antwort Bundesrat auf Anfrage 15.1073 Gross; Urteil des Bundesgerichts 6B_249/2009 vom 26. Mai 2009 E. 2.1.

36 Koller (Fn. 20), BSK-StGB I, N. 6 zu Art. 80 StGB. ständigkeit der Strafvollstreckungsbehörde. 37

\section{Verhältnis zu Art. 379 StGB}

28 Art. 379 Abs. 1 StGB räumt den Kantonen die Kompetenz ein, privaten Einrichtungen die Bewilligung $\mathrm{zu}$ erteilen, Freiheitsstrafen in der Form der Halbgefangenschaft und des Arbeitsexternats sowie Massnahmen gemäss den Art. 5961 und $63 \mathrm{zu}$ vollziehen. Diese Bewilligungskompetenz schliesst die Verwahrung gemäss Art. 64 StGB nicht mit ein. ${ }^{8} 8$

29 Art. 379 StGB steht jedoch einer gestützt auf Art. 80 StGB gewährten Platzierung von pflegebedürftigen verwahrten Person in einem privaten Wohnheim nicht entgegen. Denn Art. 379 StGB regelt die generelle Zulassung von privaten Institutionen, die unabhängig des Vorliegens besonderer Gründe, etwa derjenigen von Art. 80 Abs. 1 StGB, mit dem Vollzug von Strafen und Massnahmen betraut werden können. Demgegenüber

37 Siehe etwa Koller (Fn. 20), BSK-StGB I, N. 6 zu Art. 8o StGB; Baechtold/Weber/Hostettler (Fn. 28), S. 145 N. 62; Adrian Krähenmann/ Andreas Schweizer/ Tobias Tschumi, Hungerstreik im Strafvollzug. Drei Fragen zum Fall Bernard Rappaz, jusletter vom 10. Januar 2011, Rz. 5; Antwort Bundesrat auf Anfrage 15.1073 Gross.

38 Heinz Sutter, Kommentierte Textausgabe (Fn. 29), S. 227; Trechsel/Lieber, PK (Fn. 14), N. 1 zu Art. 379 StGB; Martino Imperatori, in: Niggli/Wiprächtiger (Hrsg.), Basler Kommentar zum Strafrecht II, Art. 111-392 StGB, 3. Aufl., Basel 2013, N. 2 zu Art. 379 StGB; Baechtold/Weber/Hostettler (Fn. 28), S. 72 N. 15; Benjamin Brägger, Der Verwahrungsvollzug in der Schweiz im Lichte der neueren Rechtsprechung des Europäischen Gerichtshofs für Menschenrechte und des deutschen Bundesverfassungsgerichts, in: Queloz/Noll/von Mandach/Delgrande (Hrsg.), Überwachen und Strafen: Neuere Entwicklungen im Justizvollzug, Bern 2018, S. 121-144, S. 129. Unter altem Recht bestand diese Möglichkeit auch für Verwahrungen. 
regelt Art. 80 Abs. 1 StGB die Abweichung von den allgemeinen Vollzugsregeln im Einzelfall. Art. 80 StGB geht dabei von der Person und deren besonderen Bedürfnissen aus, während Art. 379 StGB die generelle Zulassung bestimmter Privatanstalten regelt, die nicht auf Ausnahmefälle beschränkt ist.

Wenn die Voraussetzungen von Art. 80 Abs. 1 StGB erfüllt sind, geht Art. 80 StGB der Bestimmung von Art. 379 StGB vor. Wenn eine abweichende Vollzugsform gemäss Art. 80 Abs. 1 StGB aufgrund der besonderen Pflegebedürftigkeit geboten ist, spielt es keine Rolle, ob der betreffenden Institution eine generelle Bewilligung zum Vollzug der entsprechenden Strafsanktion erteilt worden ist bzw. erteilt werden kann. Die einzelfallbezogene Regelung von Art. 80 StGB geht der allgemeineren Organisationsbzw. Kompetenznorm von Art. 379 StGB vor.

Dem Umstand, dass Art. 379 StGB bei der generellen Zulassung privater Anstalten den Vollzug von Verwahrungen gemäss Art. 64 StGB ausnimmt, ist bei der Auslegung von Art. 80 StGB dadurch Rechnung zu tragen, dass auch für den begründeten Einzelfall eine Subsidiarität privater Anstalten gegenüber staatlichen Institutionen besteht. Demgemäss muss also immer zuerst versucht werden, eine angemessene Betreuung in staatlichen Institutionen zu gewährleisten, bevor die Einweisung in ein privates Wohnheim erfolgen darf.

\section{Verhältnis zu aktuellen parlamentarischen Vorstössen}

32 Aktuelle parlamentarische Vorstösse zum Verwahrungsvollzug39 stehen der hier vorgeschlagenen Lösung nicht entgegen: Es ist zwar nicht auszuschliessen, dass diese Vorstösse allgemein $\mathrm{zu}$ einer restriktiveren Verwahrungspraxis führen werden. Nach wie vor müsste aber aus grundrechtlicher Sicht gewährleistet sein, dass besonders pflegebedürftige verwahrte Personen unter angemessenen Bedingungen untergebracht werden, nötigenfalls in einem privaten Wohnheim, wenn - und solange - keine anderen der Pflegebedürftigkeit angemessenen (staatlichen) Vollzugsinstitutionen zur Verfügung stehen. Eine restriktivere Verwahrungspraxis als Folge aktueller politischer Vorstösse dürfte die in der Einleitung dargestellten Herausforderungen weiter verschärfen und der Bedarf nach Lösungen für besonders pflegebedürftige verwahrte Personen dadurch evidenter werden.

\section{Fazit und Diskussion}

33 Zusammenfassend kann festgehalten werden, dass ein grundrechtlicher Anspruch besonders pflegebedürftiger

$39 \overline{\text { Interpellation } 18.3123 \text { Addor (Ist es nicht an der }}$ Zeit, die Initiative für die lebenslange Verwahrung gefährlicher Straftäter tatsächlich umzusetzen?); Motion 17.3572 Guhl (Längeres Prüfungsintervall nach drei negativen Prüfungen der Verwahrung); Anfrage 15.1073 Gross (Schwerkranke in schweizerischen Gefängnissen); Postulat 16.3915 Rickli (Überprüfung der Entlassung von Tätern aus dem Straf- und Massnahmenvollzug bei Gefährlichkeit und Rückfallgefahr); Postulat 13.3978 Rickli (Verwahrungspraxis in der Schweiz); Motion 16.3002 Kommission für Rechtsfragen Nationalrat (Einheitliche Bestimmungen zum Strafvollzug bei gefährlichen Tätern); Motion 11.3767 Rickli (Keine Hafturlaube und Ausgänge für Verwahrte). 
verwahrter Personen auf Vollzugsmodalitäten besteht, die ihrer besonderen Pflegebedürftigkeit angepasst sind. Da weder auf Bundesebene noch auf kantonaler Ebene ausdrückliche Rechtsgrundlagen für eine Platzierung von besonders pflegebedürftigen verwahrten Personen in privaten Wohnheimen bestehen, muss der Weg über eine Auslegung verwandter Bestimmungen führen.

34 Die Lösung ist u.E. in der analogen Anwendung von Art. 8o StGB zu finden. Für eine besonders pflegebedürftige verwahrte Person darf in analoger Anwendung von Art. 80 Abs. 1 lit. a StGB von den Vollzugsregeln abgewichen werden, wenn damit der Pflegebedürftigkeit im Einzelfall entsprochen werden kann. Wenn die erforderliche Pflege nicht in einer herkömmlichen, staatlichen Strafanstalt bzw. Massnahmenvollzugseinrichtung realisiert werden kann, kann die verwahrte Person für den weiteren Vollzug der Verwahrung in ein Wohnheim eingewiesen werden. Dabei kann es sich entsprechend der weiten Auslegung der «geeigneten Einrichtung» in Art. 80 Abs. 2 StGB auch um eine private Institution handeln, wenn die im Einzelfall notwendigen Sicherheitsvorkehrungen in der privaten Pflegeinstitution getroffen werden können.

35 Die Platzierung von besonders pflegebedürftigen verwahrten Personen in privaten Wohnheimen ist demnach an mehrere (kumulative) Voraussetzungen geknüpft:

36 Erstens muss die Pflegebedürftigkeit i.S.v. Art. 80 Abs. 1 lit. a StGB einen ausgeprägten Schweregrad aufweisen und (voraussichtlich) über eine längere
Dauer anhalten. Die Pflegebedürftigkeit darf also bspw. nicht mit kürzeren Behandlungen in externen Institutionen oder anderen Vorkehren behoben werden können.

37 Zweitens darf eine pflegebedürftige verwahrte Personen nur in ein privates Wohnheim eingewiesen werden, wenn der besonderen Pflegebedürftigkeit im regulären Justizvollzug nicht angemessen begegnet werden kann und auch keine anderen staatlichen Vollzugsinstitutionen zur Verfügung stehen, in denen an die besondere Pflegebedürftigkeit angepasste Vollzugsbedingungen gewährleistet werden könnten. In diesem Zusammenhang ist anzumerken, dass der Gesetzeswortlaut von Art. 80 Abs. 1 StGB («[...] darf zugunsten des Gefangenen abgewichen werden[...]») zwar ein Ermessen der zuständigen Behörde zum Ausdruck bringt, sich aus dem grundrechtlich Anspruch der Betroffenen jedoch eine Verpflichtung des Staates ergibt, besonders pflegebedürftige verwahrte Personen unter angepassten Haftbedingungen zu platzieren und insbesondere die notwendige Pflegebetreuung zu erbringen. Der Staat ist demnach trotz der «Darf-Formulierung» von Art. 80 StGB verpflichtet, Vollzugsorte mit angepassten Vollzugsbedingungen zur Verfügung zu halten (vgl. auch Art. 377 Abs. 2 StGB).40 Daraus folgt weiter, dass die zuständige Behörde nicht

40 $\overline{\text { Es bestehen bekanntlich bereits einzelne derarti- }}$ ge Abteilungen und weitere sind geplant. Dazu etwa Amt für Justizvollzug Zürich (Fn. 1), S. 48 ff.; Graf (Fn. 1), S. 138; Folco Galli, Einen altersgerechten Vollzugsplatz anbieten. Die Seniorenabteilung der JVA Lenzburg stellt sich den Herausforderungen der Zeit, in: info bulletin 2/2016, S. 13-16, $13 \mathrm{ff}$. 
nur dazu berechtigt ${ }^{41}$, sondern dazu verpflichtet ist, besonders pflegebedürftige verwahrte Person in einem privaten Wohnheim zu platzieren, wenn etwa aus organisatorischen, logistischen oder baulichen Gründen 42 im regulären Vollzug keine angemessenen Vollzugsbedingungen geschaffen werden können 43 und keine andere staatliche Institution mit entsprechenden Angeboten verfügbar ist.44 Umgekehrt ist u.E. klar, dass private Wohnheime, zumindest ohne entsprechende Vereinbarungen mit den Kantonen, nicht verpflichtet sein können, pflegebedürftige verwahrte Personen aufzunehmen.

38 Drittens muss das private Wohnheim die durch Bundesrecht und kantonales Recht vorgegebenen Anforderungen erfüllen. 45 Dazu gehören unter anderem Sicherheitsanforderungen, die dem aktuellen Flucht- und Gefährdungsrisiko entsprechen müssen. ${ }^{6}$ Die hier vorgenommene Auslegung von Art. 80 StGB bedeutet mithin auch nicht, dass das öffentliche Interesse an der Sicherung der verwahr-

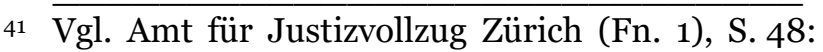
«Sollte der politische Wille für ein justizeigenes Alters- und Pflegeheim fehlen, besteht auch die Möglichkeit, alte Gefangene in entsprechend gesicherten Alters- und Pflegeheimen unterzubringen, welche durch Private geführt werden.»

42 Dazu etwa Hostettler/Marti/Richter (Fn. 3), S. 4 ff. und 9; Graf (Fn. 1), S. 136; Galli (Fn. 40), S. 15; Graber/Hotz/Holenstein (Fn. 1), S. 755 f.

43 Bspw. durch die Anstellung von Pflegepersonal durch die Justizvollzugsanstalt oder durch den Einbezug von externem Pflegepersonal (bspw. Spitex, Pflegepersonal aus kooperierendem Alters-/Pflegeheim etc.).

44 Vgl. auch Amt für Justizvollzug Zürich (Fn. 1), S. 59 .

45 Dazu Graf (Fn. 1), S. 139 f.; Amt für Justizvollzug Zürich (Fn. 1), S. 59 ff.; vgl. etwa auch Urteil des Bundesgerichts 6B_1198/2016 vom 29. Juni 2017.

46 Vgl. Graf (Fn. 1), S. 136: "Alte Menschen werden tendenziell übermässig gesichert, sind also unrechtmässig untergebracht.» ten Person zugunsten derer pflegerischer Betreuung vernachlässigt wird. Sicherheitsrisiken kann bei einer Platzierung von verwahrten Personen mit entsprechenden Vorkehren genauso begegnet werden, wie bei als abweichende Vollzugsform praktizierten Einweisungen in Wohnheime im Rahmen des Vollzugs von Freiheitsstrafen oder therapeutischen Massnahmen. Die dazu bestehende Rechtsprechung des Bundesgerichts ist analog $\mathrm{zu}$ übernehmen. 47 Die für die Anordnung der abweichenden Vollzugsformen zuständige Behörde hat bei ihrem Entscheid und insbesondere bei der konkreten Ausgestaltung der alternativen Unterbringung die Schutzinteressen der Allgemeinheit, des Personals und der Mitbewohner angemessen zu berücksichtigen. 48

$39 \mathrm{Zu}$ diesen drei Voraussetzungen müssen eventuell zusätzlich formelle Voraussetzungen erfüllt sein, sofern eine derartige Platzierung gleichbedeutend ist mit einer Vollzugslockerung. Namentlich besteht in diesen Fällen gemäss Art. 90 Abs. $4^{\text {bis }}$ i.V.m. Art. 75a StGB die Option der Fallvorlage an die Fachkommission, die auf kantonaler oder konkordatlicher Ebene als Vorlagepflicht ausgestaltet sein kann.

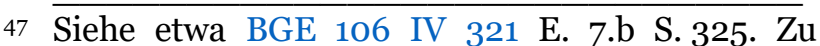
dieser Rechtsprechung etwa Viredaz/Vallotton (Fn. 35), CR, N. 6 zu Art. 80 StGB.

48 Siehe etwa Koller (Fn. 20), BSK-StGB I, N. 12 zu Art. 8o StGB. 